\title{
Automatic Welding System Using Speed Controllable Autonomous Mobile Robot*
}

\author{
Taewon $\mathrm{KIM}^{* *}$, Takeshi SUTO**, Junya KOBAYASHI**, \\ Jongcheol KIM** and Yasuo SUGA***
}

\begin{abstract}
A prototype of autonomous mobile robot with two vision sensors for automatic welding of steel plates was constructed. The robot can move straight, steer and turn around the robot center by controlling the driving speed of the two wheels respectively. At the tip of the movable arm, two CCD cameras are fixed. A local camera observes the welding line near the welding torch and another wide camera observes relatively wide area in front of the welding part. The robot controls the traveling speed in accordance with the shape of the welding line. In the case of straight welding line, the speed of the robot is accelerated and the welding efficiency is improved. However, if the robot finds a corner of welding line, the speed is decelerated in order to realize the precise seam tracking and stable welding. Therefore, the robot can realize precise and high speed seam-tracking by controlling the travel speed. The effectiveness of the control system is confirmed by welding experiments.
\end{abstract}

Key Words: Welding Robot, Autonomous Mobile Robot, Vision Sensor, Speed Control, Seam-Tracking

\section{Introduction}

In recent years, autonomous mobile robots for welding of relatively large constructions have been studied to increase the efficiency and flexibility of the welding proc$\mathrm{ess}^{(1)-(4)}$. One of the authors had already proposed an autonomous mobile welding robot with vision sensor, and effectiveness of the robot system for steel plate welding was shown ${ }^{(4)}$. However, the welding precision was inferior because of the vibration and instability of the robot movement. In particular, if the welding line has curved line or corner, precise control is necessary to track accurately the welding line and to obtain sound welded joint. Accordingly, in this study, an intelligent autonomous mobile robot for welding with two cameras, which can monitor the shape and position of the welding line to be welded, is developed. The robot can change the welding speed according to the shape of the welding line. That is to say, if the welding line is straight, the robot moves in high speed. However, if the robot approaches to a corner, the welding

* Received 25th June, 2005 (No. 05-4196)

** Graduate Student of Keio University, 3-14-1 Hiyoshi, Kouhoku-ku, Yokohama 223-8522, Japan

*** Department of Mechanical Engineering, Keio University, 3-14-1 Hiyoshi, Kouhoku-ku, Yokohama 223-8522, Japan. E-mail: suga@mech.keio.ac.jp speed decreases to track precisely the welding line. In this paper, the welding robot system and effectiveness of this system are discussed.

\section{Robot System}

Figure 1 shows the setup of the welding robot system developed in this study. The system is constructed mainly by a mobile robot body with two cameras and a TIG welding torch, a welding power source, an image monitor, an image processing board $(512 \times 440$ pixels, 8 bit $)$, two personal computers (CPU: 1.2 MHz) and etc. The two computers are used for controlling robot movement and con-

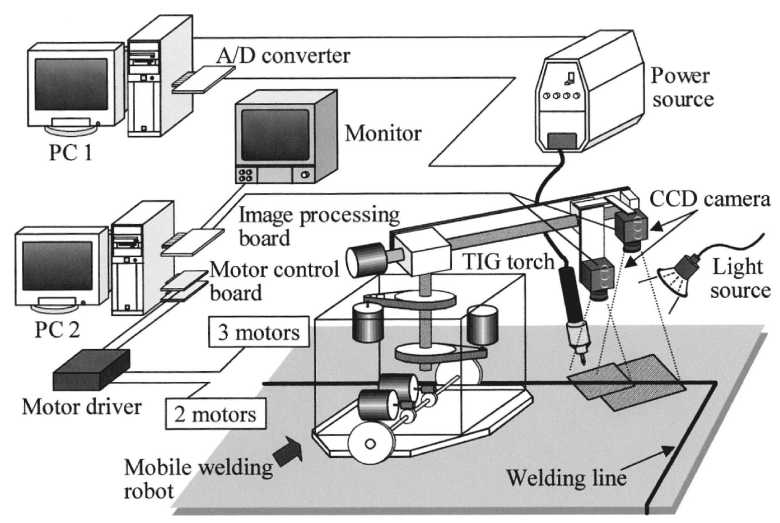

Fig. 1 Setup of autonomous mobile welding robot 


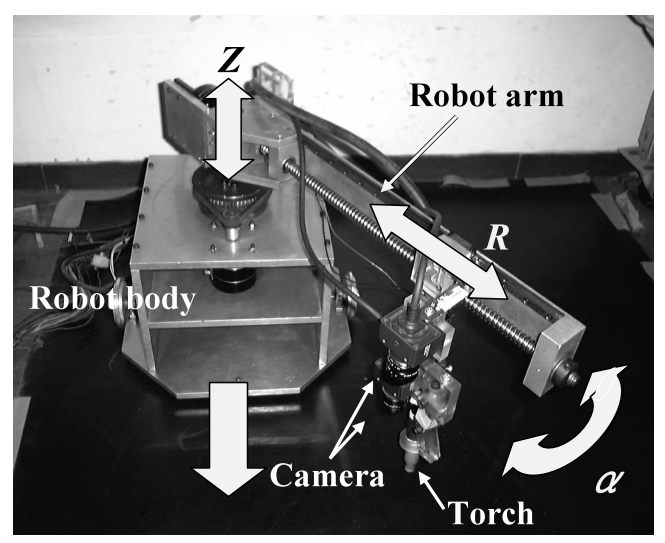

Fig. 2 Appearance of the robot

trolling welding conditions respectively.

Figure 2 shows the appearance of the robot. The robot has two driving wheels and two casters (2DW2C type). The robot can move straight, steer and turn around the robot center by controlling the driving speed of these two wheels respectively.

Furthermore, it has a movable arm as shown in Fig. 1. The arm has $R-\alpha-Z$ coordinates, and two CCD cameras and a welding torch are fixed on it as shown in Fig. 2. The cameras and the torch can move along the $R$ direction on the robot arm. The wide camera has resolution of $0.46 \mathrm{~mm} / \mathrm{pixel}$, and it monitors the shape of the welding line that lies in front of the robot. Another local camera has resolution of $0.12 \mathrm{~mm} / \mathrm{pixel}$, and it detects the position of welding line just in front of the welding torch in order to realize the precise seam tracking.

The image data acquired by the cameras are taken into the personal computer through an image processing board, and then image processing is performed. Using the processed image data, control of seam tacking, welding speed control and control of welding conditions are executed.

\section{Monitoring and Tracking of Welding Line}

\subsection{Image processing for line detection}

Generally, since the brightness of the welding line is lower than the surface of base metal, it is relatively easy to discriminate the welding line from surroundings. However, miss-detection caused by noise in the images sometimes occurs in welding by the conventional method in which welding line is detected using scanning lines. Therefore, in this study, binarization and Hough transform are used to detect welding line as stated later. The algorithm of the image processing used in this system is as follows:

(1) Binarization: Figure 3 (a) shows an original image with a welding line acquired by the CCD camera. In order to discriminate the welding line from background, image processing of the binarization was applied. For the determination of the threshold value, p-tile method was

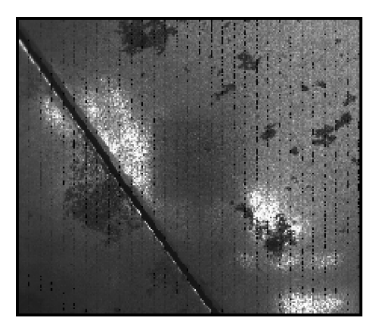

(a) Original

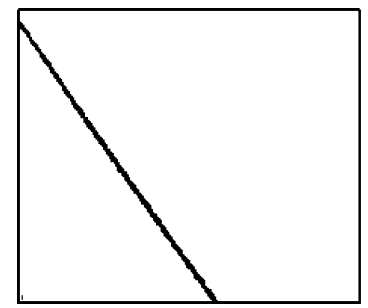

(c) Noise elimination

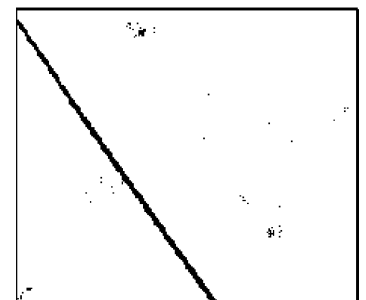

(b) Binarization

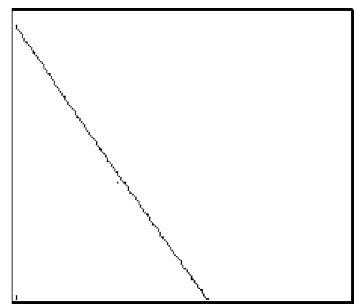

(d) Thinning
Fig. 3 Image processing for extraction of welding line

used, in which the value was selected using area of the dark pixels. In this system, the area of the welding line was estimated by its length and thickness. Binary image obtained is shown in Fig. 3 (b).

( 2 ) Noise elimination: As the binary image includes noise, image processing of labeling and elimination of small area was applied in order to eliminate noise. Resultant image is shown in Fig. 3 (c).

(3) Thinning: Welding line has some thickness in a binary image. Since the tip of the welding torch should trace the center of the line, image processing of thinning was applied to the binary image. Thinning is also effective in decrease of image noise. Figure 3(d) shows an image obtained by thinning.

\subsection{Hough transform using local polar coordinates}

Generally, a welding line is constructed by some continuous lines. In this system, Hough transform was used to abstract stably the welding line from a noisy image. Transformation from $x-y$ Cartesian coordinates to $\rho-\theta$ coordinates is expressed by the following formula.

$$
\rho=x \cos \theta+y \sin \theta
$$

Where $\rho$ and $\theta$ are parameters, and the former is length of normal line from original point to a line and the latter angle between the line and $x$-axis. However, the method has a problem that it is necessary relatively long calculation time and large computer memory. Therefore, Hough transform using local polar coordinates was proposed in order to decrease the calculation time for line detection.

Figure 4 shows the principle of the Hough transform using local polar coordinates. A line in the $x-y$ coordinates is expressed by the following formula.

$$
\theta=\beta
$$

Where $\beta$ is a inclination angle of the line in local po- 


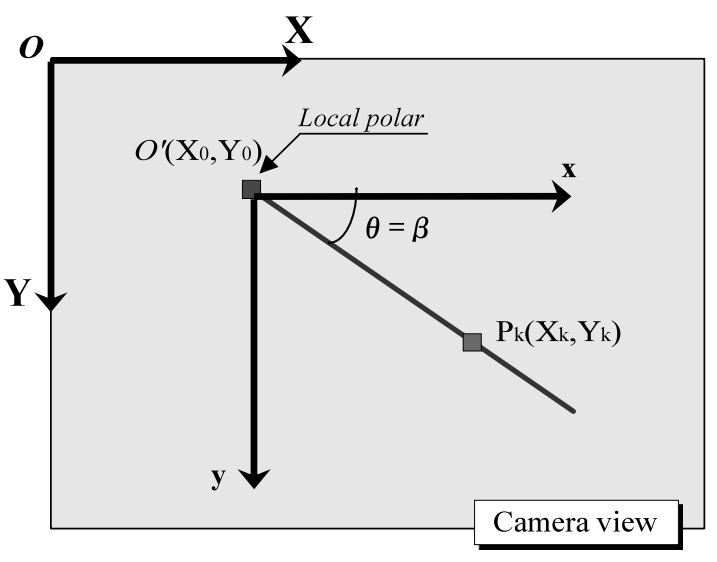

Fig. 4 Hough transform in local polar coordinates

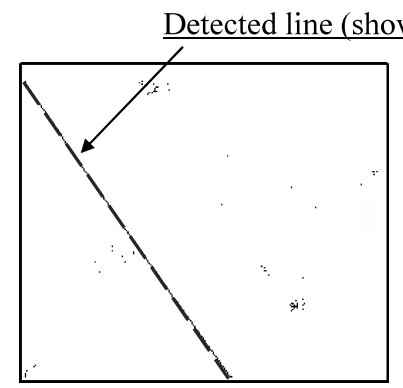

(a) Detected line

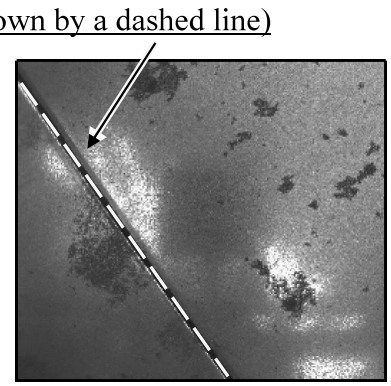

(b) Line superimposed on the original image
Fig. 5 Abstracted image of welding line

lar coordinates with the original point, $O^{\prime}$. If many feature points exist on this direction, it can be recognized that these feature points construct a linear line. If a feature point has a coordinate of $P_{k}\left(X_{0}, Y_{0}\right)$ on the $x-y$ space, the inclination angle of the line, $\theta$, is expressed by the following formula.

$$
\begin{aligned}
& \theta=\tan ^{-1}\left(\left(Y_{k}-Y_{0}\right) /\left(X_{k}-X_{0}\right)\right) \quad\left(X_{k} \neq X_{0}\right) \\
& \theta=\pi / 2 \quad\left(X_{k}=X_{0}\right)
\end{aligned}
$$

The value of the angle $\theta$ is voted into $U[\theta]$ array.

These processes are performed from 0 to 180 degree of inclination angle. If the inclination angle, $\theta$, coincides with the direction of the line, number of the vote will indicate the maximum value. As a result, a linear line is detected by relatively less calculation, and high speed processing can be realized. Welding line detected by the Hough transform is shown in Fig. 5. In the figure, (a) shows a detected line indicated by a dashed line and (b) the line superimposed on the original image shown in Fig. 3 (a).

In the case of line detection using Hough transform, the selection of the threshold of vote number, $T v$, is important. In particular, when the welding line has a corner as shown in Fig. 6(a), the distance between the welding point and the corner becomes shorter in accordance with the progress of welding. Therefore, the threshold value,

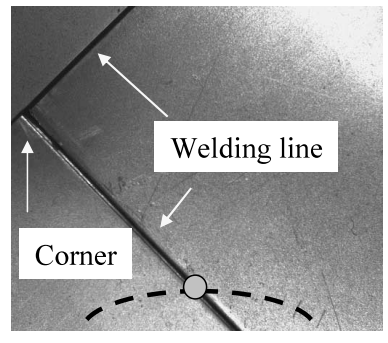

(a) Image of welding line with a corner

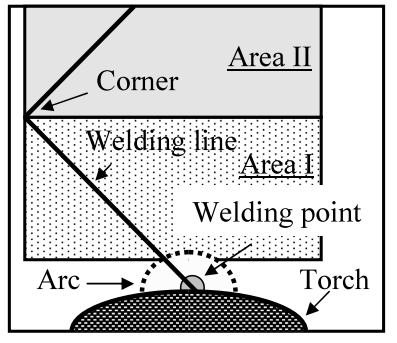

(b) Division of image into two area to detect welding line with a corner
Fig. 6 Abstraction of welding line with corner

$T v$, should change in accordance with this length of welding line. In order to detect always the welding line stably, following method was proposed. First of all, if a corner is recognized in front of the welding point, the position of the corner is detected. Then the image is divided into two local areas at the corner as shown in Fig. 6 (b). Then, Hough transform is applied to two areas respectively. In this case, the $T v$ value is decided considering the welding line length in each local area.

\section{Welding Speed Control}

In automatic arc welding using mobile robot, the welding phenomena has tendency to be unstable due to the vibration and/or instability of robot motion. In order to realize high efficiency welding and high quality welded joint in robot welding, adaptive control of welding conditions is necessary. Generally, relatively good welds can be obtained by stable arc phenomena in welding of linear line. However, since the welding process tends to be unstable in the welding of line with corner, precise welding should be performed to obtain sound welds. Accordingly, a new welding control algorithm was proposed to realize the efficient process and high quality welded joint.

Figure 7 shows the concept of the robot control algorithm. In the figure, movement of a mobile welding robot is shown schematically. Furthermore, views of welding line acquired by local camera and wide camera are shown in it. Figure 7 (a) shows images of straight line and (b) welding line with a corner.

In welding of linear line the moving speed of the robot increases and high speed welding was performed, because the welding phenomena and robot motion are stable. On the contrary, when the visual sensor of wide camera detects a corner of welding line in front of the welding point as shown in Fig. 7 (b), the speed of the robot decreases to slower welding speed by which stable welding and smooth robot motion can be realized.

Figure 8 shows a flowchart of seam tracking algorithm. First of all, the robot begins to move and acquire two images by wide and local cameras respectively. The precise position of the welding line just in front of the 


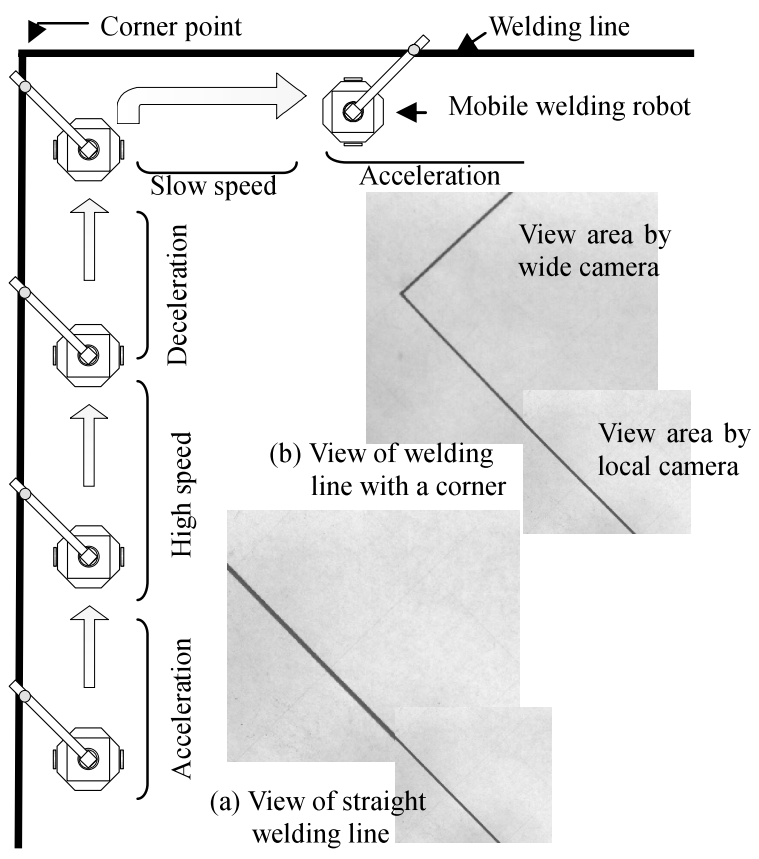

Fig. 7 Schematic illustration showing moving speed control and acquired images of welding lines

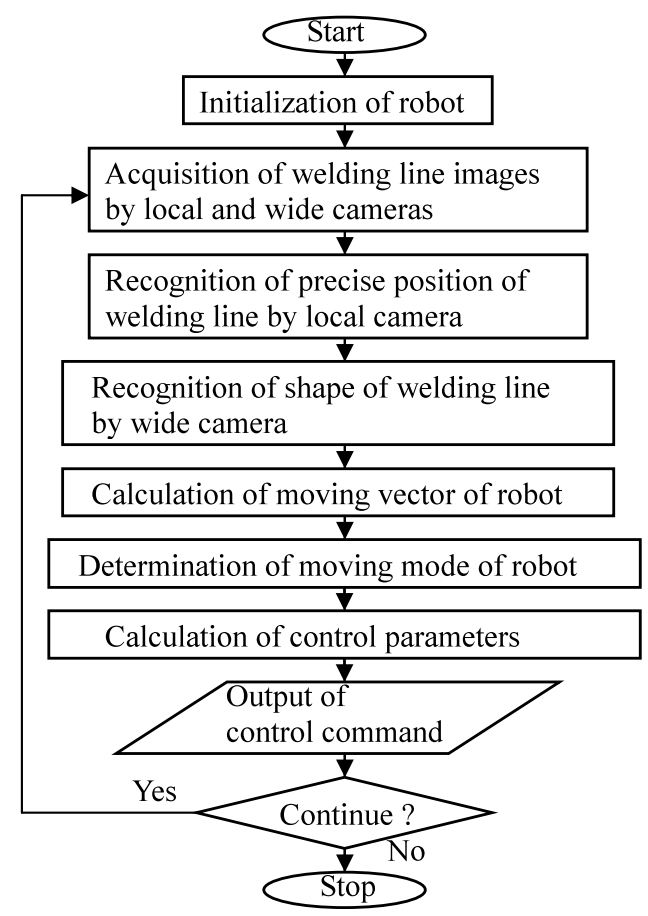

Fig. 8 Flowchart for robot control

torch is detected by the local camera, and accordingly precise seam tracking is performed. At the same time, the wide camera acquires an image of the front area, and monitors the shape of the welding line. In case the vision system recognizes that the welding line is straight, the moving speed of the robot will be increased. Therefore, high speed and efficient welding is realized. If the vision system detects a corner of welding line, the moving speed of the robot is decreased gradually, and welding is performed
Table 1 Welding conditions

\begin{tabular}{|l|l|}
\hline Welding process & TIG arc welding (DCEN) \\
Initial welding current & $80 \mathrm{~A}$ \\
Arc voltage & $9 \cdot 11 \mathrm{~V}$ \\
Arc length & $1.5 \mathrm{~mm}$ \\
Welding speed & $4-10 \mathrm{~mm} / \mathrm{s}$ \\
Shielding gas & Ar $101 / \mathrm{min}$ \\
Control cycle & $0.4 \mathrm{~s}$ \\
\hline
\end{tabular}

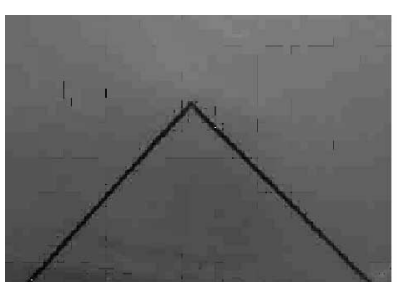

(a) Welding line

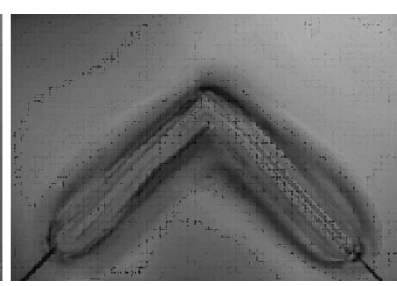

(c) Result of welding

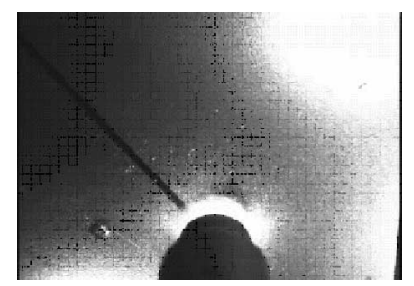

(b) TIG arc welding

Fig. 9 Result of welding experiment

in lower speed. As a result, precise seam tracking and stable welding are performed at the corner area.

When the welding speed changes in a welding process, the welding current should be changed in accordance with the speed to obtain uniform weld penetration. In this system, the welding current, It, was controlled so as to keep the weld heat input, $Q v$, constant.

\section{Result of Welding Experiment}

Table 1 shows an example of welding condition used in the welding control experiments. The welding conditions were selected by many preliminary welding experiments. As the welding process, TIG arc welding process was used. The initial welding current was $80 \mathrm{~A}$. The current was controlled in accordance with welding speed. The welding speed was changed between 4 to $10 \mathrm{~mm} / \mathrm{s}$. In welding of straight line the welding speed was kept at the maximum speed of $10 \mathrm{~mm} / \mathrm{s}$. At the corner area it was decelerated to the minimum speed of $4 \mathrm{~mm} / \mathrm{s}$. Arc length was kept at $1.5 \mathrm{~mm}$ and it is controlled by monitoring the arc voltage. In this welding experiment, the target arc voltage was set at $10 \mathrm{~V}$. The control cycle was $0.4 \mathrm{~s}$. As the base metal, SPCC mild steel plate of $1.6 \mathrm{~mm}$ in thickness was used.

Figure 9 shows the welding results, in which the welding line has a corner with angle of 90 degree. In the figure, (a) shows base metal before welding, (b) situation of TIG arc welding and (c) result of welding. From the 


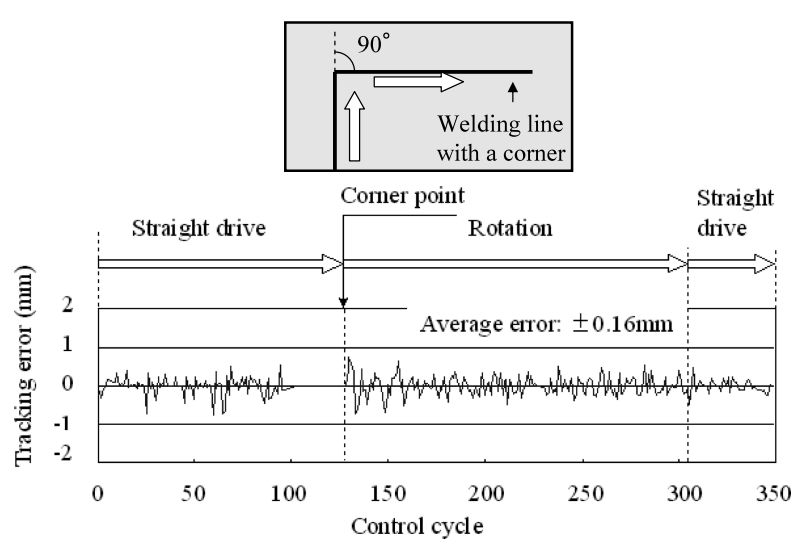

Fig. 10 Tracking error

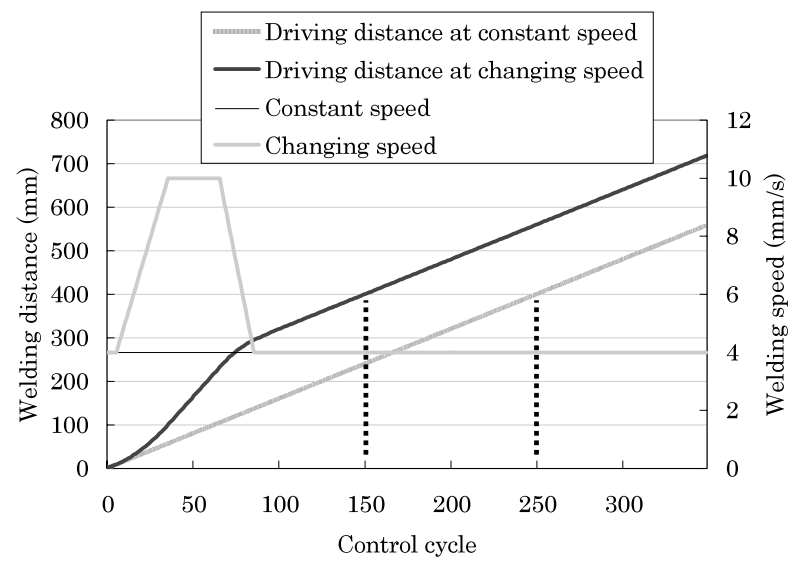

Fig. 11 Change of welding speed and moving distance in tracking of welding line with corner

photographs, it is confirmed that a sound weld is obtained using the welding robot system.

Figure 10 shows the average error of seam tracking in some moving mode of robot. First of all, the robot moves straight and then rotates near the corner. The tracking was performed along the corner with angle of 90 degree. Then it moves straight again. In the straight drive the tracking error shows about $0.12 \mathrm{~mm}$ in average. Approaching to the corner, since the tracking speed decreases, the tracking error drastically decreases. However, the robot begins to rotate, the tracking error increases again because of instable robot movement. However, in this case the tracking error is similar to that in straight drive. When the rotation finishes, the robot moves straight again and the tracking error decreases. From the figure, the average tracking error was $0.16 \mathrm{~mm}$, and the maximum error was within the target maximum error of $\pm 0.8 \mathrm{~mm}$.

Figure 11 shows change of welding speed and moving distance in progress of welding. In this case, the welding line has a corner with angle of 90 degree. From the figure, it took about 250 control cycles $(=100 \mathrm{~s})$ for welding length of $400 \mathrm{~mm}$ in a constant welding speed of $4 \mathrm{~mm} / \mathrm{s}$.

On the contrary, in case the welding speed is controlled, the welding speed increases just after the welding start. The speed accelerates up to the maximum speed of $10 \mathrm{~mm} / \mathrm{s}$ in 25 control cycles.

When the wide camera detects a corner in front area, the welding speed decreases gradually to the minimum speed of $4 \mathrm{~mm} / \mathrm{s}$. As a result, it takes about 150 control cycles $(=60 \mathrm{~s})$ for welding length of $400 \mathrm{~mm}$. In comparison between these two results, it is confirmed that the speed control system is effective in efficient welding.

As a result of this study, it is confirmed that the control system of the autonomous mobile welding robot is effective in thin metal plate welding.

\section{Conclusion}

Main results obtained by the investigation are summarized as follows.

(1) An autonomous mobile welding robot with vision sensors was made, and automatic welding control system was constructed.

(2) An algorithm to abstract the welding line image from a noisy image was proposed.

( 3 ) Image processing based on the Hough transform using local polar coordinates was proposed to detect the welding line shape and position in higher speed, and the validity of the system was confirmed.

( 4 ) Using welding speed control algorithm in automatic welding of welding line with corner, it is confirmed that precise and stable welding are performed efficiently.

( 5 ) As a result of automatic welding control experiments using the welding robot system, it was confirmed that sound welds were obtained.

\section{References}

(1) Sugitani, Y., Murayama, M. and Tamaoki, N., Lattice Welding Robot with High Speed Rotating Arc, Proc. 1st Materials and Processing Conference, M\&P '93, (in Japanese), (1993), pp.246-247.

( 2 ) Seliger, G. and Mueller, H., Prototype eines Mobilen Kleinroboters zum Schweissen von Grossauteilen aus Stahl, Schweissen \& Schneiden, Vol.47, No.9 (1995), pp.714-716.

( 3 ) Seliger, G., Hsieh, L.H. and Mueller, H., Programming and Navigation of a Mobile Welding Robot, Proc. 25th International Symposium on Industrial Robots, (1994), pp.649-654.

(4) Suga, Y., Muto, A. and Kumagai, M., Automatic Seam Tracking of Welding Line by Autonomous Mobile Robot for Plates Welding, Trans. Jpn. Soc. Mech. Eng., (in Japanese). Vol.63, No.612, C (1997), pp.342-348. 